\title{
Emerging Insights into the Moonlighting Functions and Evolutionary Origins of Mitochondrial RNA Methyltransferases
}

\author{
Sam Manna and Ashley Harman* \\ Department of Microbiology, La Trobe University, Melbourne, Victoria, Australia
}

Received: 26 November, 2014; Accepted: 20 January, 2015; Published: April 17, 2015

"Corresponding author: Ashley Harman, Department of Microbiology, La Trobe University,Melbourne, Victoria, Australia, Tel: +613-9479-2657; Fax: +613-9479-1222; E-mail: a.harman@latrobe.edu.au

Keywords: Mitochondria; RNA Methyltransferase; Pentatricopeptide repeat; Mitochondrial transcription factor

RNA methyltransferases mediate the addition of methyl groups to ribonucleotides. Such modifications have implications for the structure and function of RNA. While methylation is reported in mitochondrial RNAs, the enzymes that catalyze these reactions often remain elusive; however with advances in sequencing technologies, genomic analysis is beginning to uncover their identities. Due to the complex evolutionary history of mitochondria, little is known about the origin and function of methyltransferases. This article explores two emerging families of mitochondrial RNA methyltransferases. We discuss the evidence suggesting they possess diverse bacterial (endosymbiotic and non-endosymbiotic) origins and have acquired functions in addition to methylation.

The mitochondrial transcription factor B (mtTFB) proteins function in transcription initiation by associating with the other components of the mitochondrial transcription machinery to induce open-complex formation [1]. They also mediate the methylation of mitochondrial small subunit rRNA to facilitate ribosome assembly, stability and biogenesis [2]. The mtTFB proteins are widespread throughout eukaryotes and have arisen from a bacterial dimethyltransferase (DMT) family, which subsequently obtained a secondary function as a transcription factor.

The homology between mtTFB and an Escherichia coli rRNA adenine DMT protein (KsgA) was identified based on structural similarity. Additionally, DMTs exist throughout bacteria, eukaryotes and even archaea [2]. It is noteworthy that mtTFB homologs are more closely related to bacterial DMTs than to eukaryotic nuclear DMTs [2]. Furthermore, mtTFB is homologous to $\alpha$-proteobacterial DMTs [2,3]. Given the origins of mitochondria, it is likely that mtTFB arose from a KsgA homolog from the endosymbiont genome. This evolutionary relationship is supported by complementation studies. E. coli $\mathrm{ksgA}$ mutants lack methylated rRNA and are resistant to the aminoglycoside antibiotic kasugamycin [3,4]. The introduction of a mtTFB homolog into these mutants restores methylation and sensitivity to kasugamycin [4]. Furthermore, the methyltransferase activity of mtTFB has been implicated in aminoglycoside-induced deafness, a disease involving hypermethylation of mutated mitochondrial small subunit rRNA and increased aminoglycoside sensitivity [3]. This sensitivity is caused by an increased binding affinity for aminoglycosides to the mutated mitochondrial small subunit rRNA structure.

Metazoans possess two mtTFB homologs (TFB1M and TFB2M) as a result of gene duplication. While each human mtTFB is capable of both functions, there is a marked difference in their primary activity. TFB1M is primarily a rRNA methyltransferase whereas TFB2M is largely a transcriptional activator [2,3]. Non-metazoan eukaryotes possess one mtTFB homolog, however these proteins can mediate both rRNA methylation and transcriptional activation, as seen in the amoeboid Dictyostelium discoideum [4], or only the latter, as observed in Saccharomyces cerevisiae [3].

It is unclear how or when the DMT evolved its role in mitochondrial transcription. The apparent separation of these two functions in metazoan mtTFBs suggests that they may evolve into proteins with separate functions. Furthermore, lack of selective pressure can result in loss of methyltransferase activity, as observed in yeast where mitochondrial rRNA is no longer methylated correlating with the inability of its mtTFB to perform this function.

Similarly to mitochondrial rRNAs, mitochondrial tRNAs are also methylated and the enzymes responsible are often unknown. Proteins from the Pentatricopeptide repeat (PPR) family are characterized by a tandemly repeated 35 amino acid motif (a PPR motif) with an affinity for RNA. PPR proteins have a major role in post-transcriptional modification of organelle transcripts such as RNA processing, editing, stability, splicing and translation [5]. The identification of the pentatricopeptide repeat-containing tRNA guanine methyltransferase (PPR-TGM) class, which possess PPR motifs and a tRNA guanine methyltransferase (TGM) domain suggest PPR proteins also mediate RNA methylation [6]. 
The TGM domain in PPR-TGM proteins catalyzes the methylation of guanine in tRNA which is involved in tertiary bond formation and its structure. Cellular slime moulds and some algae possess PPR-TGM proteins that are predicted to localize to mitochondria (and in some cases chloroplasts), suggesting this modification occurs within their organelle tRNAs [6].

PPR-TGM proteins exhibit high sequence similarity to bacterial TGMs. Interestingly, it was not the TGMs from $\alpha$-proteobacteria or cyanobacteria that displayed the most similarity. This suggested that the similarity shared between PPR-TGM proteins and bacterial TGMs was not attributable to the retention of these proteins from either the mitochondrial ( $\alpha$-proteobacteria) or chloroplast (cyanobacteria) endosymbiont. Surprisingly, PPR-TGM proteins displayed most similarity to TGMs from the Chlamydiae phylum of bacteria. It is likely that a TGM existed in the endosymbiont which became obsolete, however specific eukaryotic lineages reacquired the methyltransferase from symbiotic bacteria (Chlamydiae) via horizontal gene transfer [6].

In bacteria, TGMs possess an eight amino acid sequence hypothesized to be important for RNA-binding [7,8]. This raises a question for why the Chlamydial TGM evolved PPR motifs in eukaryotic microbes. PPR-TGM proteins lack this eight amino acid sequence and so the PPR motifs may therefore be a functional alternative to the eight amino acid sequence for RNA-binding [8]. Alternatively, the presence of PPR motifs may have provided a loss of selective pressure on the eight amino acid sequence and led to its deletion. In addition, the PPR motifs may have facilitated the acquisition of a secondary function. For example, a PPRTGM protein in D. discoideum (PtcE) mediates the cleavage of mitochondrial tRNAs via the recruitment of processing machinery $[6,9]$. Therefore, the acquisition of PPR motifs may confer both an RNA-binding and a moonlighting function to PPR-TGM proteins.

Many mitochondrial proteins encoded in the nuclear genome are not of endosymbiotic origin. However, here we focused on the evolution and function of two major families of mitochondrial RNA methyltransferases, both of which originated from bacteria.These examples demonstrate that mitochondrial RNA methyltransferases were acquired either from the $\alpha$-proteobacterial endosymbiont or horizontal gene transfer from bacteria. Unlike their bacterial homologs, these proteins also have secondary functions such as transcription initiation or tRNA processing, although the purpose of this evolutionary trend is unknown. However, beyond their primary function, mitochondrial RNA methyltransferases represent an untapped resource for understanding both evolutionary and functional aspects of mitochondria.

\section{References}

1. Posse V, Hoberg E, Dierckx A, Shahzad S, Koolmeister C, Larsson N-G, et al. The amino terminal extension of mammalian mitochondrial RNA polymerase ensures promoter specific transcription initiation. Nucleic Acids Res. 2014; 42(6): 3638-3647.

2. Shutt TE, Gray MW. Homologs of mitochondrial transcription factor B, sparsely distributed within the eukaryotic radiation, are likely derived from the dimethyladenosine methyltransferase of the mitochondrial endosymbiont. Mol Biol Evol. 2006; 23(6):1169-1179.

3. Cotney J, Shadel GS. Evidence for an early gene duplication event in the evolution of the mitochondrial transcription factor B family and maintenance of rRNA methyltransferase activity in human mtTFB1 and mtTFB2. J Mol Evol. 2006; 63(5):707-717.

4. Manna S, Le P, Barth C. A unique mitochondrial transcription factor B protein in Dictyostelium discoideum. PloS One. 2013; 8(7):e70614.

5. Schmitz-Linneweber C, Small I. Pentatricopeptide repeat proteins: a socket set for organelle gene expression. Trends Plant Sci. 2008; 13(12):663-670.

6. Manna S, Barth C. Identification of a novel pentatricopeptide repeat subfamily with a C-terminal domain of bacterial origin acquired via ancient horizontal gene transfer. BMC Res Notes. 2013; 6:525. doi:10.1186/1756-0500-6-525.

7. De Bie LGS, Roovers M, Oudjama Y, Wattiez R, Tricot C, Stalon V, et al. (2003) The yggH gene of Escherichia coli encodes a tRNA (m7G46) methyltransferase. J Bacteriol. 2003; 185(10): 3238-3243.

8. Tourasse NJ, Choquet Y, Vallon O. PPR proteins of green algae. RNA Biol. 2013; 10(9): 1526-1542.

9. Manna S, Brewster J, Barth C. Identification of pentatricopeptide repeat proteins in the model organism Dictyosteliumdiscoideum. Int J Genomics. 2013; 2013: 586498. doi: 10.1155/2013/586498. 